\title{
Improved mixing rates of directed cycles by added connection
}

\author{
Balázs Gerencsér* Julien M. Hendrickx ${ }^{\dagger}$
}

October 8, 2018

\begin{abstract}
We investigate the mixing rate of a Markov chain where a combination of long distance edges and non-reversibility is introduced: as a first step, we focus here on the following graphs: starting from the cycle graph, we select random nodes and add all edges connecting them. We prove a square factor improvement of the mixing rate compared to the reversible version of the Markov chain.
\end{abstract}

Keywords: mixing rate, random graph, non-reversibility.

\section{Introduction}

We study the mixing properties of certain Markov chains which describe how fast the distribution of the state approaches the stationary distribution regardless of the initial conditions. The overall goal is to provide significant improvement of mixing by minor modifications of the Markov chain.

Mixing time and rate are fundamental quantities in the study of Markov chains 9, [12 and are the object of active research; they are also highly relevant to applications where mixing properties are strongly tied with performance metrics. This is for example the case for Markov chain Monte Carlo methods, which provide cheap approximations for integrals, and also allow sampling from complex distributions that would otherwise be hard to generate directly [7. Markov chains also provide a powerful scheme for approximating the volumes of high dimensional convex bodies [10, 11. A different application, average consensus, involves the distributed computation of the average of initial values at different agents in a multi-agent system (values which might correspond to measurements, opinions, etc.) [16. This can be achieved using a Markov chain for which the stationary distribution is uniform. The initial values can be viewed as a probability distribution scaled by a constant, and the Markov chain will approach the uniform distribution multiplied by the same constant, therefore the average of the initial values will be present at each node. Efficient consensus (and average consensus) approaches actually also play a role in several recent distributed optimization algorithms 13, 14. For all these applications good performance is crucial and it is determined by the dynamics of the underlying Markov chain. Mixing properties are formulated exactly to answer such questions, which are the topic of the current paper.

There are several ways of obtaining good mixing performance. In many applications, the graph of possible transitions is determined by the problem definition, but the specific transition probabilities can be chosen. When these are required to satisfy some strong symmetry properties (reversibility, described later in detail) choosing those to optimize the mixing rate can be formulated as an SDP problem 2, 3, which can be solved numerically using standard methods. Departing from these symmetry properties brings strong technical challenges, at the same time it can actually lead to significant improvement; the mixing time can indeed drop to its square root in some cases [4, 12. On the other hand, when it is possible to modify the

\footnotetext{
${ }^{*}$ B. Gerencsér is with MTA Alfréd Rényi Institute of Mathematics, Hungary, gerencser.balazs@renyi.mta.hu He is supported by NKFIH (National Research, Development and Innovation Office) grant PD 121107. This work has been carried out during his stay at Université catholique de Louvain, Belgium.

${ }^{\dagger} \mathrm{J} . \quad$ M. Hendrickx is with ICTEAM Institute, Université catholique de Louvain, Belgium, julien.hendrickx@uclouvain.be The work is supported by the DYSCO Network (Dynamical Systems, Control, and Optimization), funded by the Interuniversity Attraction Poles Programme, initiated by the Belgian Federal Science Policy Office, and by the Concerted Research Action (ARC) of the French Community of Belgium.
} 
graph of possible transitions, astonishing speedup can also be obtained by adding even a small number of randomly selected edges [1], [5], 8].

Our long term goal is to study the speedup that can be achieved by a combination of two a priori orthogonal transformations: (i) the addition of a small number of random edges, and (ii) the introduction of a strong non-reversibility. We start with a cycle graph of $n$ nodes, select a lower number $k$ of them to become hubs, then add extra edges between the hubs. This scheme is motivated by one of the renown models to represent Small World Networks, the Newman-Watts model [5, [15. The cycle presents a natural way of including asymmetry by introducing a drift meaning increased clockwise transition probabilities along the cycle and decreased counter-clockwise ones. At this stage the model needs three parameters to be specified: the placement of the hubs, the added interconnection structure on them, and the asymmetry introduced along the cycle.

In this paper we consider as a first step a model where hubs are chosen randomly, all edges between hubs are included and asymmetry is taken to the extreme: the Markov chain is a pure drift along the cycle taking deterministic clockwise steps, except at the hubs. To better understand the dynamics of the process, observe that the state of the Markov chain can be described by an arc (as the cycle is split by the hubs) and the position within that arc. The main challenge here is to show mixing happens both in term of arcs and in terms of positions within. We prove that this model reaches a mixing rate of $\Omega(k / n)$ (up to $\log n$ factors) if $k=n^{\sigma}$.

In comparison, if we were to put pure drift along the cycle but with equidistant hubs, we would have rapid mixing in term of the arcs (a perfect one after leaving the first arc), but no mixing at all in term of the position on the arc. Even by decreasing the drift or changing the interconnection structure, the mixing rate will remain $O\left((k / n)^{2}\right)[6$.

Furthermore, if we were to stay with the classical, symmetrical transitions along the cycle, the mixing rate will be again $O\left((k / n)^{2}\right)$ : for an arc at least $n / k$ long even the hitting time of the ends of the arc from the middle is $\Omega\left((n / k)^{2}\right)$. This holds for any hub placement and interconnection structure.

After all, we want to emphasize that a speedup with a mixing rate of $\Omega(k / n)$ is feasible only now that both random hubs and a drift along the cycle are implemented, as opposed to only one of these.

The rest of the paper is organized as follows. In Section 2 we formally describe the random graph model and Markov chain on which we focus and a proxy graph model that we will use for the analysis. In Section 3 we prove the main mixing rate result for this proxy graph model. We then translate our result to the primary graph model in Section 4 . In Section 5 simulations are presented complementing our asymptotic analytical results. We also demonstrate how the mixing rate changes when the drift is decreased for the model, suggesting that further performance improvements might be possible. We draw conclusions and outline possible future research directions in Section 6 .

\section{Graph models, Markov chains and mixing rates}

The concept of the graphs we consider is the following. We start with a cycle with $n$ nodes, and randomly select a low number of vertices, $n^{\sigma}$ out of the total of $n$ for some $0<\sigma<1$, which become hubs. Then we connect all hub nodes with each other. Let us now present the precise definitions.

Definition 1. Given $n, k \in \mathbb{Z}^{+}$we define the random graph distribution $B_{n}(k)$ as follows. Starting from a cycle graph on $n$ nodes, we randomly uniformly choose among the $k$ element subsets of edges and we remove the edges in the chosen subset. For the $i$ th remaining arcs, $1 \leq i \leq k$, we mark the clockwise endpoint as $a_{i}$ and the other end as $b_{i}$. Then we add all edges $\left(b_{i}, a_{j}\right)$, for all $1 \leq i, j \leq k$. An example is given in Figure 1 .

We are interested in the mixing behavior of Markov chains on these graphs. A Markov chain is reversible if for any edge $(u, v)$ of the graph the probability of the $u \rightarrow v$ transition is the same as the $v \rightarrow u$ transition. In this paper we go further from the comfortable domain of reversible Markov chains, let us now introduce the ones we will focus on.

Definition 2. For any graph coming from $B_{n}(k)$ we define the pure drift Markov chain as follows. Within any arc we set transition probabilities to 1 along the arc all the way from $a_{i}$ to $b_{i}$. From any $b_{i}$, we set transition probabilities to $1 / k$ on all edges towards all $a_{j}$. A part of such a chain is visualized in Figure 2 . 


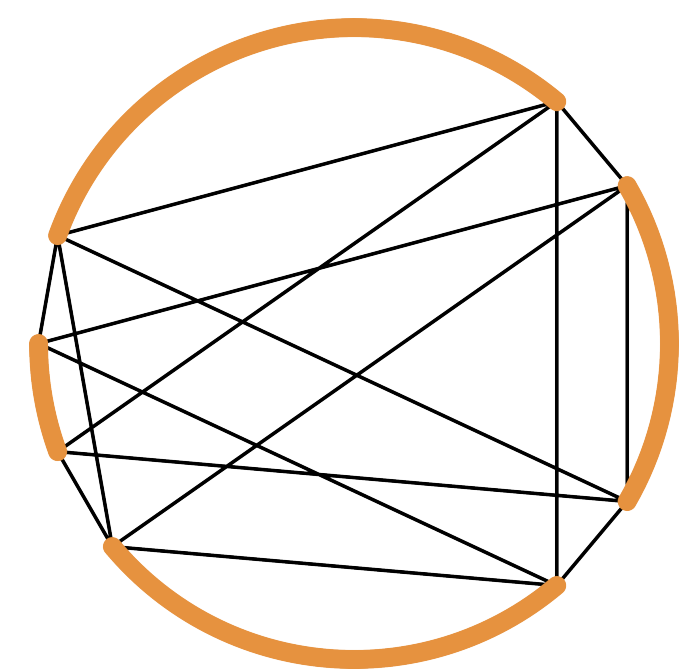

Figure 1: Example graph from $B_{n}(k)$ (see Definition 1).

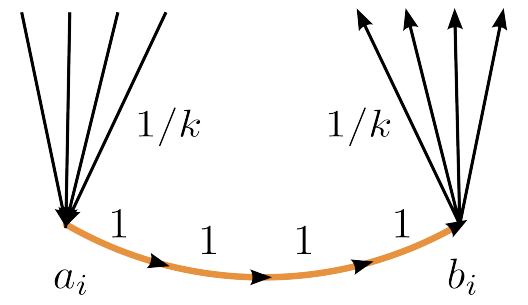

Figure 2: Example arc from the pure drift Markov chain (see Definition 2).

This is a Markov chain which has a doubly stochastic transition matrix, therefore the stationary distribution is uniform. We want to analyze the asymptotic rate as the distribution approaches the stationary distribution. This mixing performance of the Markov chain will be measured by the mixing rate:

Definition 3. For a Markov chain with transition matrix $P$ we define the mixing rate $\lambda$ as

$$
\lambda=\min \{1-|\mu|: \mu \neq 1 \text { is an eigenvalue of } P\} .
$$

Observe that for large numbers of nodes and comparably low number of hubs, arc lengths approximately follow a geometric distribution. However, they are not independently distributed, and this approximation is not valid for large lengths. Hence we introduce an alternative, simpler model of graphs reflecting approximately the same concept but technically more convenient due to the added independence. We will first establish our result for this alternative model, and then extend it in Section 4 to the original model of Definition 1 ,

Definition 4. Given $L \in(1, \infty), k \in \mathbb{Z}^{+}$, we define the random graph distribution $B(L, k)$. Let us take independently $k$ random variables

$$
L_{i} \sim \operatorname{Geo}(1 / L), \quad i=1, \ldots, k,
$$

where $\operatorname{Geo}(p)$ denotes a geometric random distribution with parameter $p$ (and 1 as the smallest possible value). We begin with a graph that is the disjoint union of $k$ arcs, paths with $L_{i}$ nodes, each of which has a "start point" $a_{i}$ and an "end point" $b_{i}$, with $a_{i}=b_{i}$ if $L_{i}=1$. Then we add all edges $\left(b_{i}, a_{j}\right)$, for $1 \leq i, j \leq k$.

The extension of the pure drift Markov chain (Definition 2) to this graph model is immediate. Note that we chose to have $L_{i}$ denote the number of nodes as opposed to the length of the path, because it leads to simpler expressions in the technical developments. 


\section{Random polynomials for pure drift Markov chains}

In order to find the mixing rate of a Markov chain, we have to know the eigenvalues of its transition matrix. For the current case we transform this eigenvalue problem into finding the roots of a certain polynomial.

Proposition 5. Let us consider the pure drift Markov chain on a random graph from $B(L, k)$. Define also

$$
q(z)=\sum_{i=1}^{k} z^{-L_{i}}
$$

Then for $\mu \neq 0, \mu \in \mathbb{C}$ is an eigenvalue of the transition matrix $P$ if and only if $q(\mu)=k$.

Proof. Assuming $\mu$ is the eigenvalue of the transition matrix $P$ let us find the corresponding eigenvector $x$. Observe that each $a_{i}$ has incoming edges from exactly the same nodes and the same weights, so the eigenvector must take the same value $x_{a_{i}}=h$ at each of them for some $h$.

For two subsequent nodes $p$ and $p^{+}$along an arc, from $x P=\mu x$ we get

$$
x_{p}=\mu x_{p^{+}}
$$

This implies that along the arcs we see the values $h, h \mu^{-1}, \ldots, h \mu^{-L_{i}+1}$ (and thus just $h$ in case of a single node arc). This already completely determines $x$ up to scaling and ensures the eigenvalue equation for all nodes except the $a_{i}$. We get a valid eigenvector if the equation also holds for $a_{i}$, which takes the form

$$
\sum_{i=1}^{k} \frac{1}{k} h \mu^{-L_{i}+1}=\mu h .
$$

Having a non-zero eigenvector implies $h \neq 0$. Therefore the above equation is equivalent to $q(\mu)=k$ if $\mu \neq 0$.

For the other direction, given a $\mu \neq 0$ such that $q(\mu)=k$, we can again build $x$ by setting $1, \mu^{-1}, \ldots, \mu^{-L_{i}+1}$ on each arc, and this will clearly be an eigenvector of $P$ with eigenvalue $\mu$.

By Definition 3, the mixing rate is high if the transition matrix $P$ has no eigenvalue near the complex unit circle. Therefore to get a lower bound on the mixing rate we have to exclude a ring shaped domain for the eigenvalues. The region to be avoided is

$$
R_{\gamma}=\left\{z: 1-\frac{1}{L \log ^{\gamma} k} \leq|z| \leq 1, z \neq 1\right\},
$$

where $\gamma$ is a constant parameter to be chosen later. We will show that asymptotically almost surely (a.a.s.) no eigenvalue of $P$ falls in $R_{\gamma}$. The width of the ring should be viewed as follows. We assume $L$ and $k$ are of similar magnitudes meaning that they have a polynomial growth rate w.r.t. each other. Therefore the width is at most a logarithmic factor lower than $1 / L$. Our key result is the following:

Theorem 6. Assume $k, L \rightarrow \infty$ while $\rho_{l}<\log L / \log k<\rho_{u}$ for some constants $0<\rho_{l}<\rho_{u}<\infty$, and fix $\gamma>4$. We use the graph model $B(L, k)$ and the definition of $q(z)$ from (1) and $R_{\gamma}$ from (2). Then for any $c, d>0$ we have

$$
P\left(\exists z \in R_{\gamma}, q(z)=k\right)=O\left(k^{-c} L^{-d}\right) .
$$

Consequently, in view of Proposition 5 and the definition of $R_{\gamma}$, we obtain for the mixing rate $\lambda>1 /\left(L \log ^{\gamma} k\right)$ a.a.s.

We show the claim in four steps. First we ensure that we can assume the $L_{i}$ variables to be bounded with high probability, this will make further estimates possible. We then check $z$ coming from different parts of $R_{\gamma}$ : we start with positive reals, then we treat complex numbers in two different ways depending on their arguments.

Intuitively the reason is the following. For some real $0<z<1, q(z)$ will be too large. Next, take $z$ with low arguments, now all $z^{-L_{i}}$ will be in the same half-plane resulting in a non-zero imaginary part for $q(z)$. 
When the argument is far enough such that $q(z)$ has a chance to have zero imaginary part again, the $z^{-L_{i}}$ will point in various different directions so that the cancellations will force the real part below $k$, a.a.s.

Now let us make all this precise. We will confirm that each of the intuitive steps above work with high probability, with the fourth requiring the $L_{i}$ to be different enough and not being extremely large. We then join these steps to give a proof of the theorem.

The probabilistic upper bound we need on the $L_{i}$ can be formulated in the following way:

Lemma 7. For any $C>1, L \geq 2$, there holds

$$
P\left(\max _{i} L_{i} \geq C L \log k\right)=O\left(k^{1-C}\right) .
$$

Proof. Assume $k \geq 2$. Remember that $L_{i}$ are i.i.d. variables with law $G e o(1 / L)$. Therefore we have that

$$
P\left(L_{i} \geq C L \log k\right)=P\left(L_{i} \geq\lceil C L \log k\rceil\right)=\left(1-\frac{1}{L}\right)^{\lceil C L \log k\rceil-1} \leq 2\left(1-\frac{1}{L}\right)^{C L \log k},
$$

based on $\left(1-\frac{1}{L}\right)^{\lceil C L \log k\rceil} \leq\left(1-\frac{1}{L}\right)^{C L \log k}$ and $\left(1-\frac{1}{L}\right)^{-1}<2$. Knowing $(1-1 / L)^{L}<1 / e$ we get

$$
P\left(L_{i} \geq C L \log k\right) \leq 2 e^{-C \log k}=2 k^{-C} .
$$

To treat all $L_{i}$ together for $1 \leq i \leq k$, we us a simple union bound.

$$
P\left(\max _{i} L_{i} \geq C L \log k\right) \leq 2 k^{1-C} .
$$

From now on, we will only investigate the (a.a.s.) event that the maximal $L_{i}$ is small as shown in Lemma 7. Let us call this event $S(C)$. We now check $z$ coming from different parts of $R_{\gamma}$. The simplest case is when $z$ is a positive real:

Lemma 8. Assume $z \in(0,1)$. Then $q(z) \neq k$.

Proof. For such $z, q(z)$ is composed of $k$ positive terms $z^{-L_{i}}$, each of them being larger than 1 because all $L_{i}$ are positive (Remember that the smallest possible value for the numbers of nodes $L_{i}$ is 1 ). Consequently the sum of the $z^{-L_{i}}$ is higher than $k$.

Next we show that there is no $z \in R_{\gamma}$ with small arguments for which $q(z)=k$.

Lemma 9. Assume $S(C)$ and take $z \in R_{\gamma}$ such that $0 \neq|\arg (z)|<\pi /(C L \log k)$. Then $\Im q(z) \neq 0$. Consequently $q(z) \neq k$.

Proof. Without loss of generality, assume $\arg (z)>0$. The event $S(C)$ ensures $0<L_{i}<C L \log k$, so the $z^{-L_{i}}$ will all be in the same half-plane, as they will have an argument in $(-\pi, 0)$. For all these values, the imaginary part is negative, and the same holds thus true for their sum. Therefore it simply cannot be 0 . in

It remains to check the elements of $R_{\gamma}$ whose argument is "large". The arguments in question are those

$$
A:=\left[\frac{\pi}{C L \log k}, 2 \pi-\frac{\pi}{C L \log k}\right] .
$$

We argue that the arguments of $z^{-L_{i}}$ become so different that strong cancellations will happen. We now formalize this idea in terms of the sum of cosines of the arguments $L_{i} x$. Note that the proposition statement uses $\cos ^{+}(y)=\max (\cos (y), 0)$ instead of simple cosines because we will need to take the sums of $\cos ^{+}$scaled by different magnitudes $\left|z^{-L_{i}}\right|$ in 114 . 
Proposition 10. Choose constants $\alpha, \beta>1$ and also $\rho_{l}, \rho_{u}$ as in Theorem 6 , and require $\rho_{l}<\log L / \log k<$ $\rho_{u}$, where $k, L, L_{i}$, defined in Definition 4, are the number of arcs, the expected arc length, and the actual lengths of the arcs. We define

$$
m=\left\lceil\log ^{\alpha} k\right\rceil, \quad \delta=\log ^{-\beta} k .
$$

Then for $k, L$ large enough we have,

$$
P\left(\sup _{x \in A} \sum_{i=1}^{m} \cos ^{+}\left(L_{i} x\right)<m-\delta^{2}\right) \geq \frac{1}{3},
$$

where $\cos ^{+}(y)=\max (\cos (y), 0)$.

Proof. Broadly speaking we will show that at least one of the $L_{i} x$ terms will be far from $2 k \pi$, which should decrease enough the sum of the cosines. For a single $x$, we state the following lemma.

Lemma 11. Use $k, L, L_{i}$ as in Definition 4. Fix $\beta>1, x \in A$ (as defined in (3)) and choose an arbitrary modulo $2 \pi$ interval $D \subset[0,2 \pi]$ of size $|D|=6 \delta=6 \log ^{-\beta} k$. Then for $k, L$ large enough we have

$$
P\left(\left\{L_{1} x\right\} \in D\right) \leq \frac{2}{3},
$$

where $\{a\}$ stands for a mod $2 \pi$.

Proof. Each element of the series $\{x\},\{2 x\},\{3 x\}, \ldots$ is either in $D$ or not. We can therefore split the series into blocks that are in $D$ and to blocks that are not. Let $t_{1}$ be the first coefficient such that $\left\{t_{1} x\right\} \in D$, then we can define the blocks

$$
\begin{aligned}
& \left\{t_{i} x\right\},\left\{\left(t_{i}+1\right) x\right\}, \ldots,\left\{\left(s_{i}-1\right) x\right\} \in D, \\
& \left\{s_{i} x\right\},\left\{\left(s_{i}+1\right) x\right\}, \ldots,\left\{\left(t_{i+1}-1\right) x\right\} \notin D,
\end{aligned}
$$

with $s_{i} \geq t_{i}+1$ and $t_{i+1} \geq s_{i}+1$. Observe that

$$
\begin{aligned}
P\left(\left\{L_{1} x\right\} \in D\right) & =\sum_{i \geq 1} P\left(L_{1} \in\left[t_{i}, s_{i}-1\right]\right) \\
& =1-\sum_{i \geq 1} P\left(L_{1} \in\left[s_{i}, t_{i+1}-1\right]\right)-P\left(L_{1}<t_{1}\right) \\
& \leq 1-\sum_{i \geq 1} P\left(L_{1} \in\left[s_{i}, t_{i+1}-1\right]\right) .
\end{aligned}
$$

We will now show

$$
P\left(L_{1} \in\left[t_{i}, s_{i}-1\right]\right) \leq 2 P\left(L_{1} \in\left[s_{i}, t_{i+1}-1\right]\right),
$$

which together with (4) will allow to conclude.

For this purpose, we first compare the number of elements in the blocks above by relating $t_{i+1}-s_{i}$ with $s_{i}-t_{i}$. We claim for $k, L$ large enough that

$$
t_{i+i}-s_{i} \geq s_{i}-t_{i}
$$

Indeed, for such $k$ we have $|D|=6 \delta<\pi / 2$. Without the loss of generality we assume $x \in[0, \pi]$.

If $s_{i}-t_{i}=1$, then we immediately get $t_{i+i}-s_{i} \geq s_{i}-t_{i}$. Otherwise, there are at least two consecutive elements of the series in $D$ and the length $6 \delta$ of $D$ is thus at least $\left(s_{i}-t_{i}-1\right) x$. Therefore we have

$$
x \leq \frac{6 \delta}{s_{i}-t_{i}-1} \leq \frac{\pi}{2\left(s_{i}-t_{i}-1\right)} .
$$

A simple consequence is $x \leq 6 \delta \leq \pi / 2$. Also, by rearranging we get

$$
x\left(s_{i}-t_{i}\right) \leq \frac{\pi}{2}+x .
$$


For the elements outside $D$, observe that since $\left(s_{i}-1\right) x$ is in $D$ and $t_{i+1} x$ is again in $D$, the $t_{i+1}-s_{i}$ intervals of length $x$ defined by $\left[\left(s_{i}-1\right) x, s_{i} x\right],\left[s_{i} x,\left(s_{i}+1\right) x\right], \ldots,\left[\left(t_{i+1}-1\right) x, t_{i+1} x\right]$ must cover at least the length of the complement of $D$, i.e. at least $2 \pi-6 \delta$. We have thus

$$
t_{i+1}-s_{i} \geq \frac{2 \pi-6 \delta}{x}-1 \geq \frac{\frac{3}{2} \pi}{x}-1 .
$$

Rearranging yields

$$
x\left(t_{i+1}-s_{i}\right) \geq \frac{3 \pi}{2}-x .
$$

We compare this with (8), note that $0<x \leq \pi / 2$ and conclude again that $t_{i+i}-s_{i} \geq s_{i}-t_{i}$.

We now show an upper bound on the block sizes. Remember that $x \in A$ defined in (3) implies $x \geq \frac{\pi}{C L \log k}$. So using (7) and $\delta=\log ^{\beta} k$, we obtain

$$
s_{i}-t_{i} \leq \frac{6 \delta}{x}+1 \leq \frac{6 \delta}{\pi / C L \log k}+1=\frac{6}{\log ^{\beta} k} \cdot \frac{C L \log k}{\pi}+1 \leq 2 C L \log ^{1-\beta} k+1 .
$$

Let us come back to the probabilities of $L_{1}$ falling within the blocks defined. As $L_{1}$ is a geometric random variable, shifting the interval of interest by $s_{i}-t_{i}$ introduces only a simple multiplicative factor:

$$
P\left(L_{1} \in\left[t_{i}, s_{i}-1\right]\right)=\left(1-\frac{1}{L}\right)^{t_{i}-s_{i}} P\left(L_{1} \in\left[s_{i}, s_{i}+\left(s_{i}-t_{i}\right)-1\right]\right) \leq \ldots
$$

We enlarge the target interval from length $s_{i}-t_{i}$ to $t_{i+1}-s_{i}$ relying on (6). Clearly by this the probability cannot decrease.

$$
\cdots \leq\left(1-\frac{1}{L}\right)^{t_{i}-s_{i}} P\left(L_{1} \in\left[s_{i}, t_{i+1}-1\right]\right) .
$$

For the coefficient at the end of $(10)$ we use $(9)$ to get for $k, L$ large enough

$$
\left(1-\frac{1}{L}\right)^{t_{i}-s_{i}} \leq\left(1-\frac{1}{L}\right)^{-2 C L \log ^{1-\beta} k-1} \leq \exp \left(3 C \log ^{1-\beta} k\right)\left(1+\frac{2}{L}\right) \leq 2 .
$$

During these estimates we used $(1-1 / L)^{-2 L} \leq \exp (3)$ and $\left(1-\frac{1}{L}\right)^{-1} \leq 1+\frac{2}{L}$ for $L$ large enough, and $\log ^{1-\beta} k$ being as close to 0 as needed for $k$ large enough.

Substituting this last bound together into 10 leads to 5 . This is enough to complete the proof as we have seen before.

To come back to the proof of Proposition 10 let us choose $D=[-3 \delta, 3 \delta]$. For small enough $\delta$, whenever we have $\left\{L_{i} x\right\} \notin D$, it implies $\cos ^{+}\left(L_{i} x\right)<1-2 \delta^{2}$. Assuming $\delta$ to be small enough is again equivalent to another (independent) bound for $k$ to be large enough. Using Lemma 11 and knowing that the $L_{i}$ are independent random variables we have

$$
P\left(\sum_{i=1}^{m} \cos ^{+}\left(L_{i} x\right) \geq m-2 \delta^{2}\right) \leq P\left(\left\{L_{i} x\right\} \in D\right)^{m} \leq\left(\frac{2}{3}\right)^{m}
$$

where $m=\log ^{\alpha} k$ was defined in the statement of the proposition. This is the type of probability bound we are looking for, but only for a single $x$. Next we extend it to all $x \in A$ simultaneously, where $A$ is the interval of interest of arguments (3). As an intermediate step, break $A$ into $\lceil A / \epsilon\rceil$ equal subintervals with $\epsilon=2 \delta^{2} /(m C L \log k)$ and choose $x_{j}$ as the middle of each of these subintervals. In this setting, no point of $A$ is further than $\epsilon / 2$ from some point $x_{j}$. Using the union bound for these chosen points we see

$$
P\left(\sup _{j} \sum_{i=1}^{m} \cos ^{+}\left(L_{i} x_{j}\right) \geq m-2 \delta^{2}\right) \leq \sum_{j} P\left(\sum_{i=1}^{m} \cos ^{+}\left(L_{i} x_{j}\right) \geq m-2 \delta^{2}\right)
$$


Taking into account the number of points $x_{j}$ in the grid and (11), we obtain

$$
\begin{gathered}
P\left(\sup _{j} \sum_{i=1}^{m} \cos ^{+}\left(L_{i} x_{j}\right) \geq m-2 \delta^{2}\right) \leq\left\lceil\frac{2 \pi m C L \log k}{2 \delta^{2}}\right\rceil\left(\frac{2}{3}\right)^{m} \\
\leq \pi\left(\log ^{\alpha} k+1\right) C L \log k \log ^{2 \beta} k\left(\frac{2}{3}\right)^{\log ^{\alpha} k}=C \pi L \log ^{2 \beta+\alpha+1} k\left(\frac{2}{3}\right)^{\log ^{\alpha} k}(1+o(1)) .
\end{gathered}
$$

In this final term $(2 / 3)^{\log ^{\alpha} k}$ decreases faster than the inverse of any polynomial in $k$ as $\alpha>1$. All others parts of the product have polynomial or lower rate in $k$. Consequently we see that the right hand side of 12 will become arbitrarily small as $k, L$ grows. In particular, it will go below $2 / 3$.

At this point we have the probability estimate for grid points $x_{j}$. We need to extend this to the complete interval $A$, introduced in (3). We show that for $k, L$ large enough we have

$$
P\left(\sup _{x \in A} \sum_{i=1}^{m} \cos ^{+}\left(L_{i} x\right) \geq m-\delta^{2}\right)<P\left(\sup _{j} \sum_{i=1}^{m} \cos ^{+}\left(L_{i} x_{j}\right) \geq m-2 \delta^{2}\right) .
$$

Indeed, for any $x \in A$ there is a grid point $x_{j}$ at most $\epsilon / 2$ away. As the derivative of $\cos ^{+}$stays within $[-1,1]$, the change of the sum when moving to $x_{j}$ from $x$ is at most

$$
\sum_{i=1}^{m} \frac{\epsilon}{2} L_{i} \leq m \frac{\delta^{2}}{m C L \log k} C L \log k=\delta^{2} .
$$

Therefore when the sum on the left hand side of $(13)$ is at least $m-\delta^{2}$ for a certain $x \in A$ then there also must be a grid point $x_{j}$ for which the sum is at least $m-2 \delta^{2}$. The inclusion of the events shows the inequality for the probabilities.

Proof of Theorem 6. Note that the right hand side of the claim can be slightly simplified. The relation between $L$ and $k$ ensures $k^{\rho_{l}}<L<k^{\rho_{u}}$ after a while, therefore the $L$ term on the right hand side can be replaced by a power of $k$. It is now sufficient to show that the probability in question is $O\left(k^{-c}\right)$ for all $c>0$, we will thus consider only this case.

Choose $C \geq c+1$. Let us assume $\max _{i} L_{i}<C L \log k$. This not being true is an exceptional event of probability $O\left(k^{-c}\right)$ as shown in Lemma 7. In order to exclude the roots from all $R_{\gamma}$, we split this region into three parts, and show that $z$ cannot be a solution of $q(z)=k$ in any of these parts.

When $0<z<1$ is a positive real, it cannot be a solution according to Lemma 8 . When $z$ has a small argument, that is, $|\arg (z)|<\pi /(C L \log k)$, we refer to Lemma 9 to confirm $z$ cannot be a solution either.

The remaining case is when $z$ has a large argument, that is, $\arg (z) \in A$. We aim to bound $\Re q(z)$. On one hand, we estimate the magnitude of the terms $z^{-L_{i}}$. Then we combine these with the cosines of the arguments to find their contributions to the real part of $q(z)$. Here we rely on Proposition 10, but let us make this precise.

We need to check the magnitude of the terms $z^{-L_{i}}$. Knowing $|z|>1-1 /\left(L \log ^{\gamma} k\right)$ and $L_{i}<C L \log k$ for $k, L$ large enough we have

$$
\begin{aligned}
|z|^{-L_{i}} & \leq\left(1-\frac{1}{L \log ^{\gamma} k}\right)^{-C L \log k} \leq\left(1+\frac{2}{L \log ^{\gamma} k}\right)^{C L \log k} \\
& \leq \exp \left(2 C \log ^{1-\gamma} k\right) \leq 1+\frac{4 C}{\log ^{\gamma-1} k} .
\end{aligned}
$$

Considering $\Re q(z)$, this gives

$$
\Re q(z)=\sum_{i=1}^{k}|z|^{-L_{i}} \cos \left(L_{i} x\right) \leq \sum_{i=1}^{k}|z|^{-L_{i}} \cos ^{+}\left(L_{i} x\right) \leq\left(1+\frac{4 C}{\log ^{\gamma-1} k}\right) \sum_{i=1}^{k} \cos ^{+}\left(L_{i} x\right) .
$$

Note that the last inequality is the reason we have been working with $\cos ^{+}$, as it would generally not hold true for cos. Let us arrange the $k$ elements of this sum into groups of $m=\log ^{\alpha} k$ arbitrary elements, 
consequently resulting in $k / m$ such groups. For a moment we assume $k$ is divisible by $m$. Let the sum of these groups be $S_{1}, S_{2}, \ldots, S_{k / m}$. According to Proposition 10 we have

$$
P\left(S_{i} \geq m-\delta^{2}\right) \leq \frac{2}{3},
$$

and each of these events are independent. Therefore the number of such events happening follows a $\operatorname{Binom}(k / m, r)$ distribution for some $r \leq 2 / 3$. From standard Chernoff bounds we see that

$$
P\left(\text { Binom }\left(\frac{k}{m}, r\right)>\frac{3 k}{4 m}\right) \leq P\left(\text { Binom }\left(\frac{k}{m}, \frac{2}{3}\right)>\frac{3 k}{4 m}\right) \leq \exp \left(-\frac{k}{96 m}\right)<k^{-c},
$$

for $k$ large enough. Consequently, at most $3 k /(4 m)$ of $S_{i}$ are larger than $m-\delta^{2}$. (with the exception of an event with probability of $O\left(k^{-c}\right)$ ). In this case we have

$$
\sum_{i=1}^{k} \cos ^{+}\left(L_{i} x\right)=\sum_{j=1}^{k / m} S_{j} \leq \frac{3 k}{4 m} m+\frac{k}{4 m}\left(m-\delta^{2}\right)=k-\frac{\delta^{2} k}{4 m} .
$$

Plugging this back into (14) we arrive at

$$
\Re q(z) \leq\left(1+\frac{4 C}{\log ^{\gamma-1} k}\right) k\left(1-\frac{\delta^{2}}{4 m}\right)=k\left(1+\frac{4 C}{\log ^{\gamma-1} k}\right)\left(1-\frac{1}{4 \log ^{2 \beta+\alpha} k}\right) .
$$

Let us choose $\gamma>2 \beta+\alpha+1$. With such a choice, the term $k$ above gets multiplied by a coefficient lower than 1 for $k$ large enough. This shows $\Re q(z)<k$ which implies $z$ is not a solution of $q(z)=k$. This a.a.s. holds simultaneously for all $z \in R_{\gamma}, \arg (z) \in A$.

For the sake of completeness, if $k$ was not divisible by $m$, we could still form $\left\lfloor\frac{k}{m}\right\rfloor$ groups as before with $\bar{k}=m\left\lfloor\frac{k}{m}\right\rfloor$ elements, then collect the sum of the remaining $k-\bar{k}$ terms into $S_{\left\lfloor\frac{k}{m}\right\rfloor+1}$. Performing the same argument and using the trivial upper bound for $\cos ^{+}$when working with $S_{\left\lfloor\frac{k}{m}\right\rfloor+1}$ we get

$$
\Re q(z) \leq \bar{k}\left(1+\frac{4 C}{\log ^{\gamma-1} k}\right)\left(1-\frac{1}{4 \log ^{2 \beta+\alpha} k}\right)+(k-\bar{k})\left(1+\frac{4 C}{\log ^{\gamma-1} k}\right) .
$$

Knowing $k-\bar{k}<m$ the new additive term compared to (15) is (poly)logarithmic, which will not compensate for the almost linear $k / \log ^{\epsilon} k$ type of loss originating from the first term. Hence once again, the right hand side will not reach $k$ and thus $z$ can not be a solution.

Regarding the parameters, previously for Proposition 10 we only needed to ensure $\alpha, \beta>1$. Therefore we can apply Proposition 10 and this reasoning for $\alpha=\beta=1+\epsilon, \gamma=4+4 \epsilon$ for any $\epsilon>0$, eventually allowing any $\gamma>4$.

Remember that during the proof, we had two small exceptional events, both having probability $O\left(k^{-c}\right)$. This allows thus confirming the theorem with the condition on $\gamma$ and with the probability bound on the exceptional cases.

Theorem 6 guarantees the absence of eigenvalues with large absolute value (except at 1) with high probability. We can reformulate it in the following way

Theorem 12. Assume $k, L \rightarrow \infty$ while $\rho_{l}<\log L / \log k<\rho_{u}$ for some constants $0<\rho_{l}<\rho_{u}<\infty$. Then for any $\gamma>4$ a.a.s. we have the following bound on the mixing rate for $B(L, k)$ :

$$
\lambda>\frac{1}{L \log ^{\gamma} k} .
$$

Proof. This lower bound is a direct consequence of Theorem 6 and the definitions of $R_{\gamma}$ and the mixing rate $\lambda$. 


\section{Mixing rates for $B_{n}(k)$}

We now translate Theorem 12 to our initial graph model $B_{n}(k)$, where the total number of nodes are fixed beforehand. For this purpose, we first show that for any $L \in \mathbb{R}^{+}$the conditional distribution of $B(L, k)$ conditioned on having $n$ nodes in total is the same as $B_{n}(k)$ in the following sense. We will use the compact notation $\left.B(L, k)\right|_{n}$ for the aforementioned conditional distribution.

We need to map a graph from $\left.B(L, k)\right|_{n}$ to the cycle. Given such a graph $G$ (as we build it in Definition 4 and $s \in\{1,2, \ldots n\}=[n]$ define $T(G, s)$ as follows. Map $a_{1}$ to node $s$, then progressing along the arc of $L_{1}$ to $s+1, s+2, \ldots$, continuing with $L_{2}, L_{3}, \ldots$ taking the numbers $s+i$ modulo $n$ once needed. Map the edges of $G$ consistently with the nodes, then $T(G, n)$ is a graph on the labeled nodes $[n]$.

Proposition 13. Given are $n, k \in \mathbb{Z}^{+}, L \in \mathbb{R}^{+}$. Let $U(n)$ denote the uniform distribution on $[n]$ and $T^{*}(\cdot, \cdot)$ the induced measure transformation of $T(\cdot, \cdot)$ defined above.

Then $T^{*}\left(\left.B(L, k)\right|_{n}, U(n)\right)=B_{n}(k)$. Simply speaking, if we randomly choose the starting point where we map $\left.B(L, k)\right|_{n}$ to the cycle, we get the distribution $B_{n}(k)$.

Note that the randomization in the mapping does not change the internal structure of the graph, therefore once the proposition is proven, we also immediately get the following:

Corollary 14. Given $n, k \in \mathbb{Z}^{+}, L \in \mathbb{R}^{+}$consider the conditional distribution $\left.B(L, k)\right|_{n}$. Then the corresponding distribution of the mixing rate $\lambda$ is the same as the distribution of $\lambda$ for $B_{n}(k)$.

Proof of Proposition 13. For any $l_{1} \geq 1$ we have $P\left(L_{1}=l_{1}\right)=(1-p)^{l_{1}-1} p$ and the arc of $a_{1} \cdots b_{1}$ will consist of $l_{1}$ nodes, (with $p=\frac{1}{L}$ ). Consequently, in the setup of $\left\{L_{1}=l_{1}, L_{2}=l_{2}, \ldots, L_{k}=l_{k}\right\}$ we get a total of $n$ nodes exactly if $\sum_{i=1}^{k} l_{i}=n$. The probability of such an instance is

$$
P\left(L_{1}=l_{1}, L_{2}=l_{2}, \ldots, L_{k}=l_{k}\right)=\prod_{i=1}^{k}(1-p)^{l_{i}-1} p=(1-p)^{\sum_{i=1}^{k} l_{i}} p^{k}=(1-p)^{n-k} p^{k} .
$$

This probability is independent of the choice of $\left\{L_{i}\right\}_{i=1}^{k}$, therefore the conditional distribution is uniform on all the possibilities.

A uniform number on $[n]$ is supplemented and we need to relate this joint variable to the distribution of $B_{n}(k)$. It is straightforward to see that $T$ will map any element in the support of $\left.B(L, k)\right|_{n} \times[n]$ to an element of the support of $B_{n}(k)$ as they are built the same way.

Moreover, this will be a homogeneous map in the sense that each element of the support of $B_{n}(k)$ will be obtained exactly $k$ times. To see this, start from any such element, we will get the $k$ different preimages depending on which arc we choose to be $L_{1}$. (Note that two subsequent deleted edges might lead to an "arc" without edges, of size 1 in our current notation.)

In the end, both the conditional distribution $\left.B(L, k)\right|_{n}$ and $U(n)$ were uniform, applying the map $T$ that is a uniform $k$-fold cover on a target space will result in a uniform distribution on its range, confirming the claim of the proposition.

Our last ingredient is a lower bound on the probability for a random graph model $B(n / k, k)$ to have exactly $n$ nodes.

Lemma 15. The probability for a random graph $B(n / k, k)$ to have exactly $n$ nodes is at least $\frac{1}{n}$.

Proof. We have seen in the proof of Proposition 13 that all instances of $n$ nodes have the same probability $\left(1-\frac{k}{n}\right)^{n-k}\left(\frac{k}{n}\right)^{k}$. Moreover, standard combinatorial arguments show that there are $\left(\begin{array}{l}n-1 \\ k-1\end{array}\right)$ ways of distributing the $n$ nodes into the $k$ arcs, leading to the following probability of obtaining exactly $n$ nodes, and event that we denote by $M(n, k)$

$$
P(M(n, k))=\left(\begin{array}{l}
n-1 \\
k-1
\end{array}\right)\left(\frac{k}{n}\right)^{k}\left(1-\frac{k}{n}\right)^{n-k}=\left(\begin{array}{l}
n-1 \\
k-1
\end{array}\right) \frac{k^{k}(n-k)^{n-k}}{n^{n}} .
$$


We develop a simple asymptotic estimate for this probability. From the Stirling formula we know that

$$
\lim _{n \rightarrow \infty} \frac{n !}{\sqrt{2 \pi n}\left(\frac{n}{e}\right)^{n}}=1 .
$$

For conciseness, we will use the $\approx$ relation if the ratio of the two quantities is 1 in the limit. In this spirit we get

$$
\left(\begin{array}{l}
n \\
k
\end{array}\right) \approx \frac{\sqrt{2 \pi n}\left(\frac{n}{e}\right)^{n}}{\sqrt{2 \pi k}\left(\frac{k}{e}\right)^{k} \sqrt{2 \pi(n-k)}\left(\frac{n-k}{e}\right)^{n-k}}=\sqrt{\frac{n}{2 \pi k(n-k)}} \frac{n^{n}}{k^{k}(n-k)^{n-k}} .
$$

Let us plug this back to 16 , while noting $\left(\begin{array}{l}n-1 \\ k-1\end{array}\right)=\frac{k}{n}\left(\begin{array}{l}n \\ k\end{array}\right)$.

$$
P(M(n, k)) \approx \frac{k}{n} \sqrt{\frac{n}{2 \pi k(n-k)}}=\sqrt{\frac{k}{2 \pi n(n-k)}} .
$$

As a very crude bound we get for $n, k$ large enough that

$$
P(M(n, k))>\frac{1}{n}
$$

We can now extend Theorem 12 to our initial model $B_{n}(k)$.

Theorem 16. Assume $n, k \rightarrow \infty$ while $\rho_{l}<\log n / \log k<\rho_{u}$ for some constants $1<\rho_{l}<\rho_{u}<\infty$. Then for any $\gamma>4$ a.a.s. we have the following bound on the mixing rate for $B_{n}(k)$ :

$$
\lambda>\frac{k}{n \log ^{\gamma} k} .
$$

Proof. We know from Theorem 6 that the probability of the polynomial $q(z)$ having a root in the forbidden ring (and thus of the mixing rate being smaller than $\left.\frac{1}{L \log ^{\gamma} k}\right)$ bounded as $O\left(L^{-2} k^{-2}\right)=O\left(n^{-2}\right)$. Hence it remains negligible with respect to the probability of $B(n / k, k)$ having exactly $n$ nodes (for $n, k$ large enough) which we have shown in Lemma 15 to be at least $1 / n$. We deduce that the mixing rate of pure drift Markov chains for the conditional random graph model $\left.B(n / k, k)\right|_{n}$ is also at least $\frac{1}{L \log ^{\gamma} k}$ a.a.s. Proposition 13 allows then concluding that the same holds for $B_{n}(k)$, which concludes the proof.

\section{Simulations}

Following the asymptotic theoretical results we perform complementing simulations to analyze the tightness of the bounds obtained. We also explore further using numerical tools for the next step of research that is not yet treated analytically.

The mixing results we have are exciting as we see a strong speedup compared to the similar reversible Markov chain with transition matrix $\tilde{P}=\left(P+P^{\top}\right) / 2$. By this we set all transition probabilities on all edges to be equal in the two directions. For this Markov chain, if the initial distribution is concentrated in the middle of the longest arc, the Central Limit Theorem ensures that even after $\Omega\left(L^{2} \log ^{2} k\right)$ steps the probability of not leaving the arc is bounded away from 0 . Consequently we get a lower bound of the same order for the mixing time and which in turn translates to the mixing rate bound

$$
\lambda<\frac{C}{L^{2} \log ^{2} k}
$$

which is a square factor worse than our new results for the non-reversible Markov chain.

Simulations are in line with the speedup we see when comparing (18) with Theorem 12 , Figure 3 is a log-log histogram showing the decrease of $\lambda$ as the node count $n$ increases. The histogram presents the 


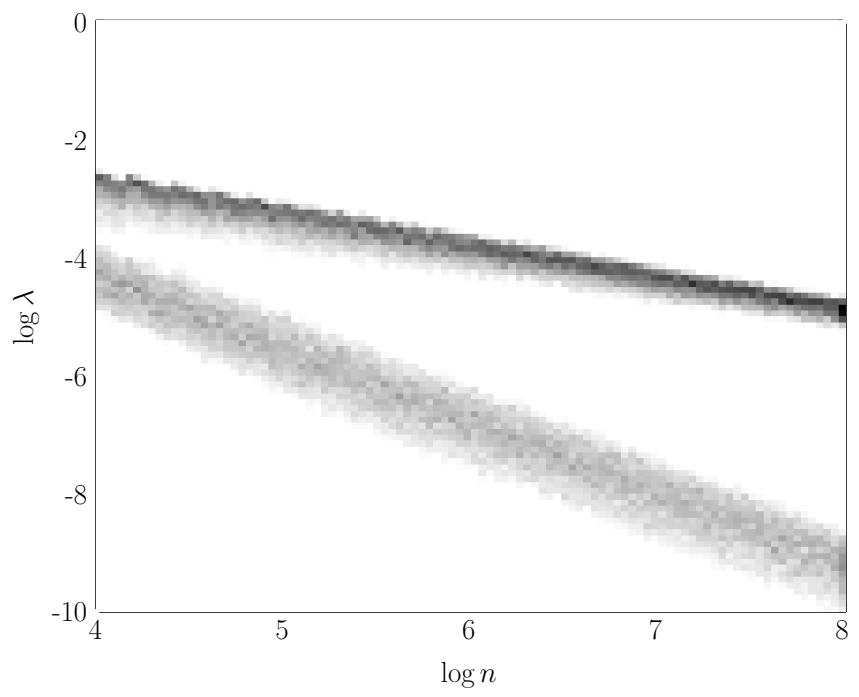

Figure 3: Histograms for the mixing rates $\lambda$ for the Markov chains on the graphs $B_{n}(\lfloor\sqrt{n}\rfloor)$. The upper stripe corresponds to non-reversible Markov chains while the lower one to the reversible variants.

simulation results for the non-reversible and reversible Markov chain and we do observe the strong separation predicted by the theoretical results. The stripe on the top presents $\lambda$ for the non-reversible Markov chains while the bottom one corresponds to the reversible ones. Figure 3 is based on 200.000 random Markov chains with $n$ ranging from 54 to 2980 and with $k=\lfloor\sqrt{n}\rfloor$. As we are interested in typical behavior of these randomized Markov chains, we discarded the top and bottom $5 \%$ of the results for every $n$ considered.

The two type of Markov chains we compared can be seen as the extremal setups: either the asymmetry is so strong that steps are deterministic along the cycle, or we have perfect symmetry. There is however a full spectrum of intermediate situations, and one may wonder which level of asymmetry is optimal. We have seen that full asymmetry is better in terms of mixing performance than full symmetry. On Figure 4 . starting from the reversible Markov chain, we gradually change the transition probabilities along the cycle until we reach the current extreme asymmetric case. Specifically, for $1 / 2 \leq q \leq 1$ we set the transition matrix $P_{q}=q P+(1-q) P^{\top}$ and compute the mixing rate of the resulting Markov chain. Here we have $P_{1 / 2}=\tilde{P}, P_{1}=P$ as expected. We perform simulations for $B_{500}(10)$ and $B_{500}(50)$. In both case, 8000 random graphs were generated and the mixing rates were computed for all graphs and for $q$ moving along $[1 / 2,1]$. Again, the top and bottom $5 \%$ were discarded. The means of the resulting mixing rates are presented in Figure 4 together with the sample standard deviations. The figures show that the optimal choice is near the extremal non-reversible case, confirming our concept. Still, interestingly a minor offset towards the reversible version still increases the mixing rate. Intuitively the two effects of the modification match well: the small loss in the speed of moving along the cycle is well compensated by the local mixing introduced.

The analytic treatment of the intermediate Markov chains for $q \neq 1 / 2,1$ brings new challenges as the non-reversible feature is still present while we lose the deterministic nature of the movement along the cycle.

\section{Conclusions}

We have seen in Theorem 12 and Theorem 16 that for both models $B_{n}(k)$ and $B(L, k)$ the mixing rate of the non-reversible Markov chain considered is much higher than the similar reversible one. The results confirm that the simultaneous application of adding long distance edges and also setting the Markov chain to be non-reversible dramatically improves the mixing rate. We believe this phenomenon is promising and could provide similar speedup effects for other reference graphs, other methods to add random edges and other means of introducing non-reversibility. 


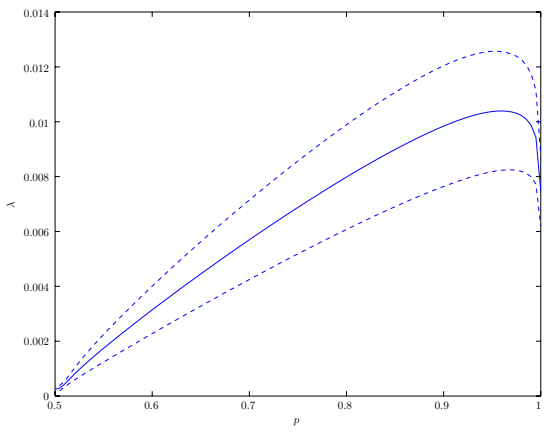

(a) Using graphs $B_{500}(10)$

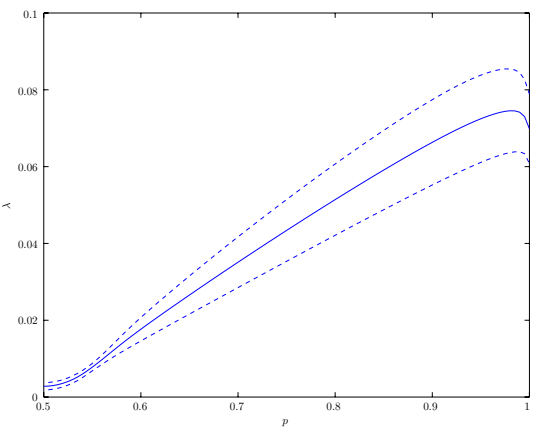

(b) Using graphs $B_{500}(50)$

Figure 4: Mixing rates of the transition matrices $P_{q}=q P+(1-q) P^{\top}$ for the interpolation between the pure drift $(q=1)$ Markov chain and its reversible version $(q=0.5)$. Solid lines follow the means while dashed lines indicate the sample standard deviations around the means.

We have also seen numerically in Figure 4 that being fully non-reversible is not necessarily optimal in this context, even though it is significantly better than being fully reversible.

Therefore one of the open questions is to find the optimal Markov chain among the intermediate cases. Another goal for future research is to consider the more realistic situation where the hubs do not have such high number of connection, for instance, by replacing the complete graph on the selected $c n^{\sigma}$ nodes with a random matching on them. Simulations similar to Figure 3 in [6] suggest that a similar speedup is to be expected.

\section{References}

[1] L. Addario-Berry and T. Lei, The mixing time of the Newman-Watts small world, in Proceedings of the Twenty-Third Annual ACM-SIAM Symposium on Discrete Algorithms, SIAM, 18 Jan 2012, pp. $1661-1668$.

[2] S. Boyd, P. Diaconis, P. Parrilo, and L. XiaO, Fastest mixing Markov chain on graphs with symmetries, SIAM J. Optim., 20 (2009), pp. 792-819.

[3] S. Boyd, P. Diaconis, And L. XiaO, Fastest mixing Markov chain on a graph, SIAM Rev., 46 (2004), pp. 667-689 (electronic).

[4] P. Diaconis, S. Holmes, And R. M. Neal, Analysis of a nonreversible Markov chain sampler, Ann. Appl. Probab., 10 (2000), pp. 726-752.

[5] R. DurRett, Random Graph Dynamics, Cambridge University Press, 2006.

[6] B. Gerencsér, Mixing times of Markov chains on a cycle with additional long range connections. arXiv:1401.1692, 2014.

[7] M. Jerrum, Mathematical foundations of the Markov chain Monte Carlo method, in Probabilistic methods for algorithmic discrete mathematics, vol. 16 of Algorithms and Combinatorics, Springer, 1998, pp. 116-165.

[8] M. Krivelevich, D. Reichman, and W. Samotiu, Smoothed analysis on connected graphs, SiAM J. Discrete Math., 29 (2015), pp. 1654-1669.

[9] D. Levin, Y. Peres, And E. Wilmer, Markov chains and mixing times, American Mathematical Society, 2009. 
[10] L. Lovász and S. Vempala, Hit-and-run from a corner, SIAM Journal on Computing, 35 (2006), pp. 985-1005.

[11] Simulated annealing in convex bodies and an $O^{*}\left(n^{4}\right)$ volume algorithm, Journal of Computer and System Sciences, 72 (2006), pp. 392-417.

[12] R. Montenegro and P. Tetali, Mathematical aspects of mixing times in Markov chains, Foundations and Trends® in Theoretical Computer Science, 1 (2006), pp. 237-354.

[13] A. Nedić And A. Olshevsky, Distributed optimization over time-varying directed graphs, IEEE Transactions on Automatic Control, 60 (2015), pp. 601-615.

[14] A. Nedić, A. Ozdaglar, And P. A. Parrilo, Constrained consensus and optimization in multi-agent networks, IEEE Transactions on Automatic Control, 55 (2010), pp. 922-938.

[15] M. Newman, C. Moore, And D. Watts, Mean-field solution of the small-world network model, Phys. Rev. Lett., 84 (2000), pp. 3201-3204.

[16] A. Olshevsky AND J. N. Tsitsiklis, Convergence speed in distributed consensus and averaging, SIAM J. Control Optim., 48 (2009), pp. 33-55. 